Lecture Notes in Computational Vision and Biomechanics 34

João Manuel R. S. Tavares

Renato Manuel Natal Jorge Editors
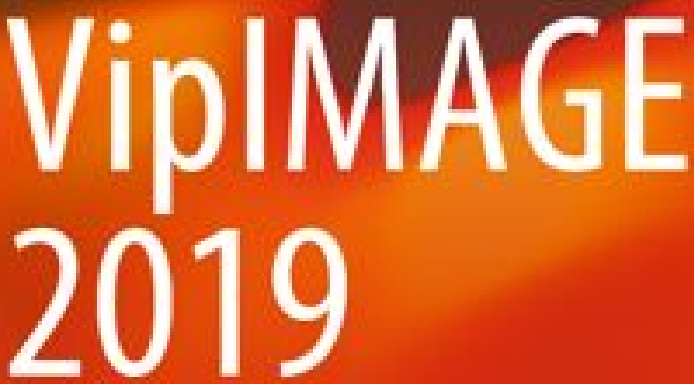

Proceedings of the VII ECCOMAS

Thematic Conference on Computational Vision and Medical Image Processing, October 16-18, 2019, Porto, Portugal

Q Springer 


\title{
Numerical characterization of a hyperelastic material to shear stress
}

\author{
Andrews V. Souza1, João E. Ribeiro², Fernando Araújo ${ }^{3}$ \\ 1 Instituto Politécnico de Bragança, Portugal, email: andrews.va.souza@alunos.ipb.pt \\ 2 Instituto Politécnico de Bragança, CIMO, Portugal, email: jribeiro@ipb.pt \\ 3 Federal Center of Technological Education of Rio de Janeiro CEFET/RJ Campus \\ Angra dos Reis, email: Fernando.araujo@cefet-rj.br
}

\begin{abstract}
In last years, some studies have been proved that there is an association between the wall shear stress with intracranial aneurysm rupture, however, is very difficult to understand the mechanical tissue behaviour when subjected to shear stresses. In this work, it is implemented numerical simulations to characterise the polydimethylsiloxane (PDMS) material when it is subjected to a shear solicitation. For this, it was initially necessary to perform some experimental tests to characterize the mechanical behaviour of the material. Based on these results, several numerical simulations were performed with the most common constitutive models in the simulation of hyperelastic materials by varying numerical factors and parameters of the numerical models.
\end{abstract}

Key-words: numerical simulation, shear stress, hyperelastic material, PDMS, Ansys ${ }^{\circledR}$.

\section{Introduction}

Some biological tissues, like soft tissues, are composed by several layers with different compositions and they are known to support large reversible deformations, also called hyperelastic behaviour. It is considered that exist four typical soft tissues: muscular tissue, neuronal tissue, epithelial tissue and neuronal tissue [1]. The mechanical behaviour can be described by hyperelastic constitutive equations or models. The hyperelastic constitutive models can be anisotropic or isotropic and it is, generally, expressed in terms of strain components or strain invariants [2]. In last decades have been developed materials with hyperelastic behaviour applied in biomedicine [3] some of them are used as prosthesis [4] other are used as in vitro models to study and analyse some pathologies [5]. So, one the most popular hyperelastic materials used in the biomedical industry is the elastomer polydimethylsiloxane (PDMS). The most important reasons of the PDMS popularity is related with its biocompatibility and biomechanical behaviour, similar to biological tissues, with applications in the study of aneurysm behaviour [6] and 
devices such as: micro pumps, optical systems, microfluidic devices [7], among others.

PDMS belongs to the group of siloxanes, however, in its advent, it was called silicocetones or silicones, but since as there was no double bond of $\mathrm{Si}=\mathrm{S}$, its name was later replaced by a specific nomenclature and its basic unit has become known as siloxanes. The most known material of this group is PDMS, a synthetic polymer whose main axis is made from the repetition of silicon and oxygen bonds and methyl groups [8]. PDMS is a material that has good microstructural characteristics, good manufacturing ability and a low cost. In addition, PDMS is thermally stable, optically transparent [9], works as a thermal and electrical insulation [10], has good chemical stability and degrades quickly in the natural environment when compared to other polymers, and it presents no environmental problem. However, the main disadvantage from the biomedical point of view is the difficulty of wetting its surface with aqueous solvents.

Many researchers have been observed that there is an association between the wall shear stress with intracranial aneurysm rupture [11]. Hemodynamics plays a central role throughout intracranial aneurysm natural history, and shear stress has emerged as an important determinant of arterial physiological characteristics [12]. However, the analysis of wall shear stress in vivo is very difficult, being in vitro solution a valid and interesting approach. In this sense, the use of PDMS to create an aneurysm model have been developed in last years by the scientific community $[13,14]$. For this reason, it is very important to understand the mechanical behaviour of this material when subjected to shear stresses. So, in the present work we have carried out numerical simulations to analyse the shear stress field in the PDMS. The numerical analysis was based on a finite element method, a computational technique that, due to the development of robust and optimized algorithms, allows simulations with high accuracy and precision results. The numerical method used in the present study, allows to test the characteristics of previously known hyperelastic materials by using mathematical models suitable for these kind of materials.

\section{Numerical simulation}

Hyperelastic models have been widely used to model the nonlinear and anisotropic behaviour of materials, since these under large deformations often recover their elasticity. The constitutive behaviour of hyperelastic materials is defined in terms of energy potential. Among all constitutive models for hyperelastic materials, in this work was used only the most common to simulate the mechanical behaviour of PDMS, which are the Mooney-Rivlin, Ogden and Yeoh $[15,16]$. However, its formulations need constants and coefficients that can be determined by fitting a suitable experimental stress-strain curve. This curve was obtained from tensile test of a PDMS specimen. In Fig 1 is possible to observe the stress-strain curve 
implemented with a specimen of Sylgard 184, which the geometry and dimensions were in agreement with the BS 2782 standard.

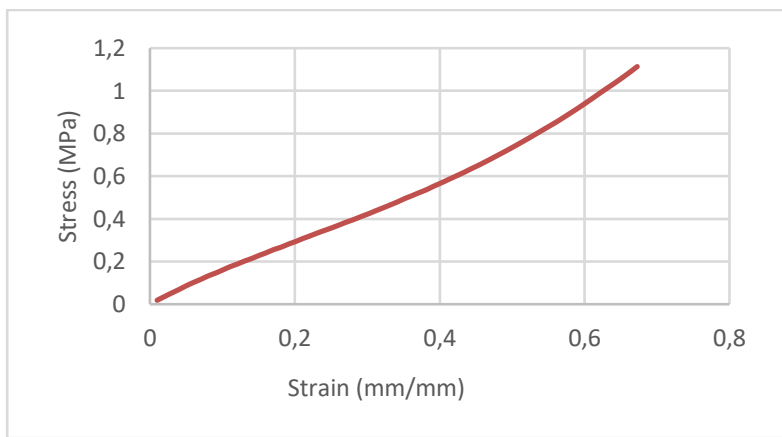

Fig. 1 Stress-strain curve of PDMS (Sylgard 184) specimen.

One the most commons tests to analyse and evaluate the shear stress is implemented by using a single lap joint. In this case, the single lap joint was used to transfer the loading from the substrate to the PDMS. In this joint, the material of adherent was the 6061 aluminium alloy and for the adhesive was the PDMS. The geometry and dimensions of the joint have been chosen and determined based on ASTM D100210 standard and can be seen in Fig 2 .

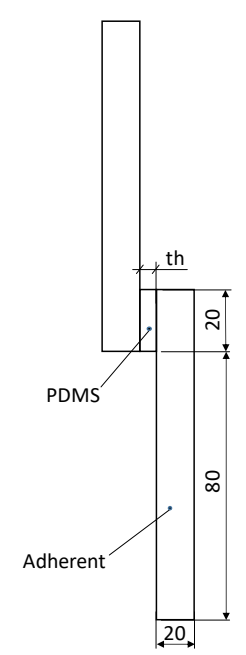

Fig. 2 Dimensions and geometry of the single lap joint.

The most important goals of this study are to analyse the influence of PDMS thickness and the applied displacement in the PDMS shear stress distribution. For this reason, was implemented nine different numerical simulations in agreement with the Table 1. 
Table 1 Simulation variables.

\begin{tabular}{|c|c|c|}
\hline Simulation & PDMS thickness [mm] & Applied displacement [mm] \\
\hline 1 & 2 & 2 \\
\hline 2 & 2 & 3 \\
\hline 3 & 2 & 4 \\
\hline 4 & 4 & 2 \\
\hline 5 & 4 & 3 \\
\hline 6 & 4 & 4 \\
\hline 7 & 6 & 2 \\
\hline 8 & 6 & 3 \\
\hline 9 & 6 & 4 \\
\hline
\end{tabular}

The numerical simulation was implemented using a commercial finite element method (FEM) software ANSYS ${ }^{\circ}$.

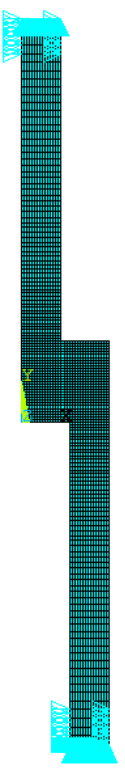

Fig. 3 Finite element mesh and boundary conditions.

To perform the numerical simulation, it was necessary to create a model with a geometry similar to that of the specimen and boundary conditions matching the experimental testing and to discretize the domain finite element mesh. For the 
material properties, a nonlinear hyperelastic behaviour, based on the constitutive models of Mooney-Rivlin, Yeoh and Ogden, was considered. The application of these models required the determination of several constants, which were identified from the experimental curve of the tensile test. Nevertheless, the adherents were considered isotropic with a linear elastic behaviour which the mechanical properties are: E=70 GPa and a Poisson's ratio of 0.3. The common nodes (adherent and PDMS) are the same, so, the properties are the average of both materials.

A bi-dimensional finite-element mesh, with 2790 parametric structural solid elements (PLANE183) was used and is shown in Fig 3. In relation to the boundary conditions of the numerical model, a uniform displacement was applied to the upper lips, stretching the single lap joint sample (Fig 3). The simulations were carried out for different values of displacement, according to Table 1.

\section{Results}

The numerical simulations implemented in this work allow to analyse the shear stress variation on the PDMS for different conditions (Table 1) and constitutive models. In Fig. 4 is represented, as an example, the results for the simulation number nine and Ogden constitutive model.

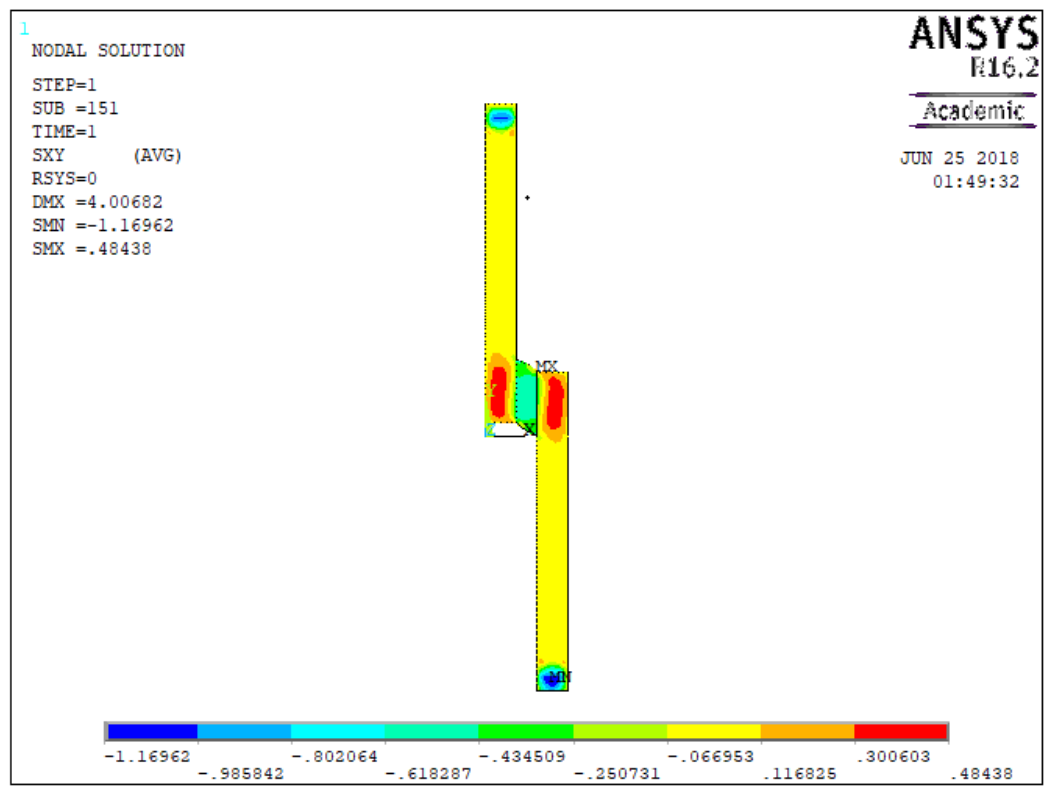

Fig. 4 Shear stress map obtained numerically for the condition 9: 6mm of PDMS, $4 \mathrm{~mm}$ of applied displacement; and the Ogden constitutive model. 
To compare the evolution of shear stresses for the different simulations it is more suitable and intuitive to define a path on the centre of the PDMS plate. In Fig. 5 is presented the chosen path to compare the obtained results. This region was chosen because is expected that the shear stress reaches the highest values.

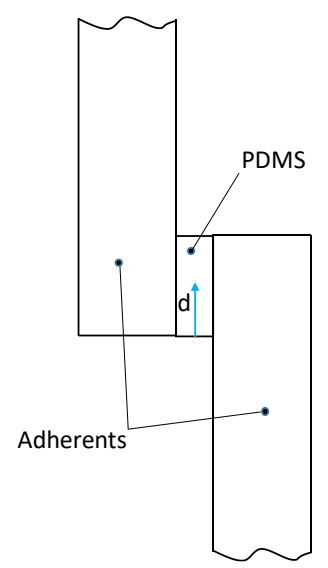

Fig. 5 The chosen path or distance (d) to analyse the evolution of shear stress on the centre of PDMD plate.

Despite all the 27 simulations had been implemented, the authors will analyse in this paper the simulations $1,3,4,6,7$ and 9 with three constitutive models. These chosen simulations constitute the situations with the highest amplitude between the variables, for example, in the simulations 1 and 3, the PDMS thickness is the same $(2 \mathrm{~mm})$, but the applied displacement are the minimum $(2 \mathrm{~mm})$ and the maximum $(4 \mathrm{~mm})$, the same principle has been used for the other analysed simulations.

In Fig. 6 and 7, is possible to observe the shear stress variation along to the chosen path, defined in Fig. 5 by the $d$ direction for the three constitutive models.

Observing both figures (6 and 7) it is verified that the constitutive models have a strong influence in the obtained results. There isn't any case in which the shear stress is the same for different constitutive models. This difference is higher when the applied displacement is higher, particularly, when is associated with the lower values of thickness. In Fig. 7 (a) it is verified that the value of shear stress for the Yeoh is, approximately, the double of the results obtained by the simulation implemented with Ogden, i.e., -4.5 MPa and -9 MPa, respectively. A similar event happens in the Fig. 7 (b). Is, also, observed in Fig. 6, that the values of shear stress obtained with Yeoh simulation are always lower than those obtained by the constitutive model of Mooney-Rivlen and the Ogden is between the two. However, in Fig. 7, it does not happen the same rule as in the previous case, so, in Fig. 7 (a) the maximum values of shear stress happed with the Yeoh and the minimum with the Ogden simulation, but, for the Fig. 7 (b) the maximum value occurs for MooneyRivlen constitutive model and the minimum values comes up for the simulation 
implemented with the Yeoh model, although, in Fig. 7 (c) is possible to observe that behaviour is also different from the Fig. 7 (a).

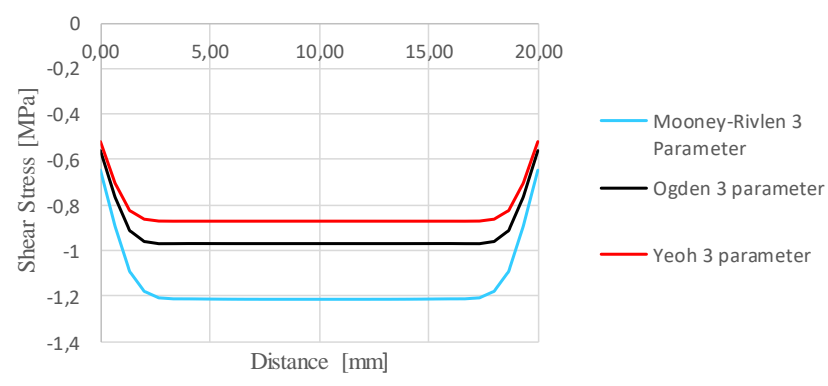

(a)

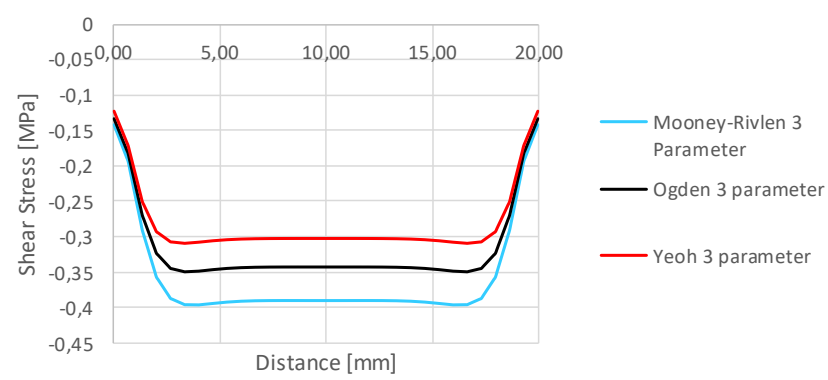

(b)

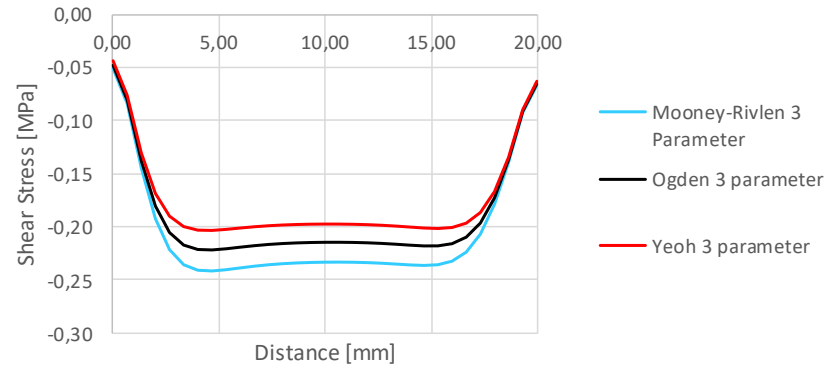

(c)

Fig. 6 Shear stress variation on PDMS along $d$ direction (distance) for simulations: (a) 1, (b) 4 and (c) 7 .

Analysing the influence of PDMS thickness and applied displacement in the shear stress, is possible to verify that for the same displacement the maximum shear stress decreases when the thickness increases. In Fig. 6, considering the Mooney-Rivlen numerical simulation, the shear stress goes from $-1.2 \mathrm{MPa}$, for $2 \mathrm{~mm}$ of PDMD thickness, to $-0.24 \mathrm{MPa}$ when the thickness rises to $6 \mathrm{~mm}$. The same phenomenon 
is verified in Fig. 7, the maximum shear stress decrease, proximally, 15 times when the PDMS thickness grow 3 times.

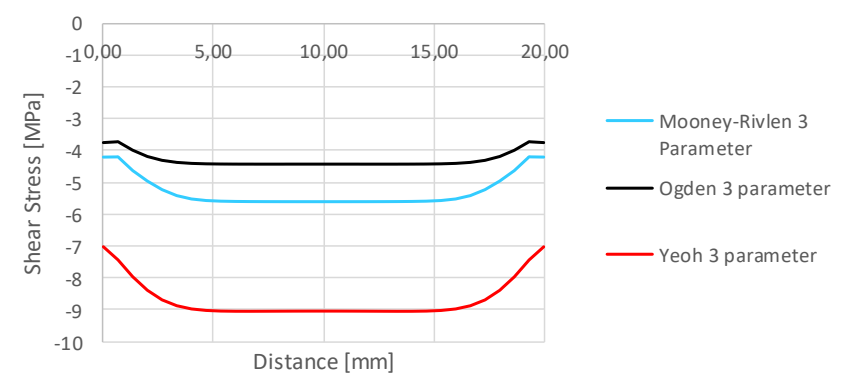

(a)

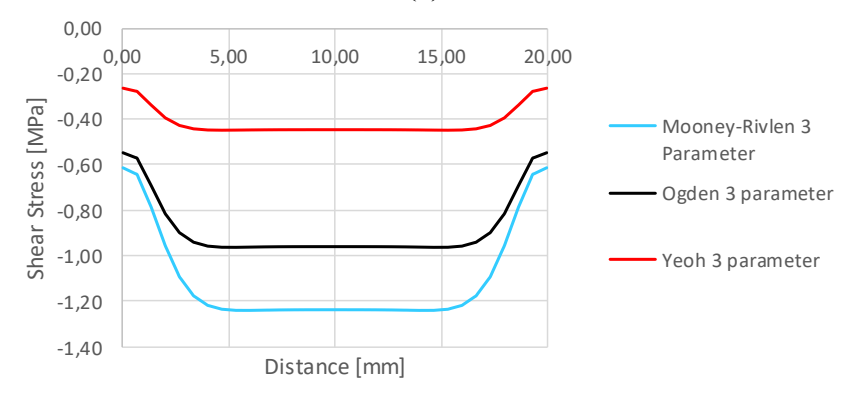

(b)

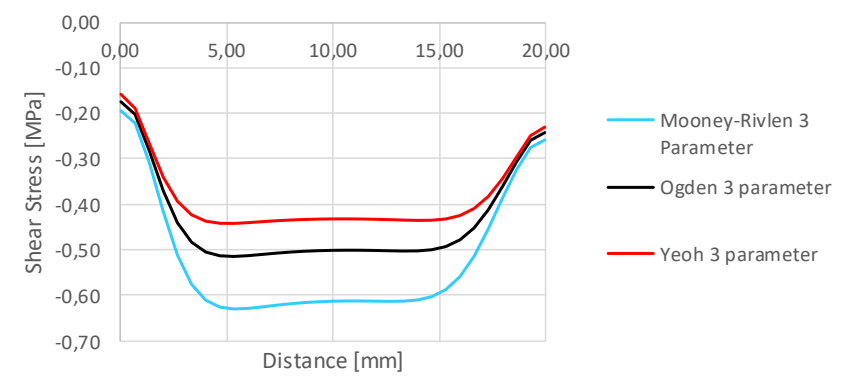

(c)

Fig. 7 Shear stress variation on PDMS along $d$ direction (distance) for simulations: (a) 3, (b) 6 and (c) 9 .

Naturally, with the increase of applied displacement the shear stress also grows. Comparing the values show in Fig. 6 (a) and Fig. 7 (a), where the PDMS thickness is same $(2 \mathrm{~mm})$ and the displacement is $2 \mathrm{~mm}$ and $6 \mathrm{~mm}$, respectively, the maximum shear stress increases from -1.2 MPa to $-9 \mathrm{MPa}$. This behaviour is repeated for the other values of thickness. 


\section{Conclusions}

The numerical simulations presented in this work were implemented with the commercial element finite code ANSYS ${ }^{\circledR}$. The main goals of this work were achieved, i.e, the simulations allow to analyse the influence of thickness and applied displacement in the shear stress for hyperelastic material (PDMS). To implemented these numerical simulations were used three constitutive models: Mooney-Rivlen, Ogden and Yeoh.

For each constitutive model was obtained a different result of shear stress. In the majority of simulations results the constitute model of Mooney-Rivlen is the most conservative, as such the highest values of shear stresses were obtained with it and the maximum values of shear stresses are among $-0.25 \mathrm{MPa}$ and $-1.25 \mathrm{MPa}$, depending of simulation variables. On the other hand, the last conservative is the Yeoh, the numerical simulations with this constitutive models result in smallest values of shear stress, from $-0.2 \mathrm{MPa}$ to $-0.9 \mathrm{MPa}$. The only exception to this rule happened with the simulation 3 , where the maximum value of $-9 \mathrm{MPa}$ was obtained with Yeoh model and the minimum (-4.2 MPa) was computed with the constitutive model of Ogden.

The influence of the PDMS thickness and applied displacement in the shear stress, was observed that for the same displacement, the maximum shear stress decreases when the thickness increases. However, for the same PDMS thickness when the applied displacement increases the shear stress also raises.

\section{Acknowledgments}

The authors gratefully acknowledge the funding by PORTUGAL2020: SI I\&DT Individual - Call 16/SI/2015 (Proj. n ${ }^{\circ}$ 9703): "Automatização de Processos de Soldadura de Estruturas Hiperestáticas em Ligas de Alumínio (APSEHAL)".

\section{References}

[1] Marieb EG, Hoehn K, (2010) Human Anatomy \& Physiology. Pearson Education, Upper Saddle River.

[2] Boyce MC, Arruda EM (2000) Constitutive models of rubber elasticity: a review. Rubber Chem. Technol. 73: 504-523

[3] Kanyanta V, Ivankovi A (2010) Mechanical characterization of polyurethane elastomer for biomedical applications. Journal of the Mechanical Behavior of Biomedical Materials, Issue 1. 3: 51-62

[4] Garcia-Gonzalez D, Garzon-Hernandez S, A. Arias (2018) A new constitutive model for polymeric matrices: Application to biomedical materials. Composites Part B: Engineering, 139: $117-129$ 
[5] Rodrigues RO, Pinho D, Bento D, Lima R, Ribeiro J (2016) Wall expansion assessment of an intracranial aneurysm model by a 3D Digital Image Correlation System. Measurement 88: 262270

[6] Pinho D, Bento D, Ribeiro J, Lima R, Vaz M (2015) An in vitro experimental evaluation of the displacement field in an intracranial aneurysm model. in: P. Flores, F. Viadero (Eds.), New Trends in Mechanism and Machine Science, Mechanisms and Machine Science, Springer 24: 261-268

[7] Faustino V, Catarino SO, Lima R, Minas G (2016) Biomedical microfluidic devices by using low-cost fabrication techniques: A review. Journal of Biomechanics, Issue 11, 49: 2280-2292

[8] Kuncová-Kallio J, Kallio PJ (2006) PDMS and its suitability for analytical microfluidic devices. Annu. Int. Conf. IEEE Eng. Med. Biol. - Proc., pp. 2486-2489

[9] Martin S, Bhushan B (2017) Transparent, wear-resistant, superhydrophobic and superoleophobic poly(dimethylsiloxane) (PDMS) surfaces”, J. Coll. Inter. Sci. 488: 118-126

[10] Cherney EA (2005) Silicone rubber dielectrics modified by inorganic fillers for outdoor high voltage insulation applications. IEEE Trans. Dielectr. Electr. Insul 12: 1108-1115

[11] Zhou G, Zhu Y, Yin Y, Su M, Li M (2017) Association of wall shear stress with intracranial aneurysm rupture: systematic review and meta-analysis. Scientific Reports, Issue 1, 7: 5331

[12] Xiang J, Tutino VM, Snyder KV, Meng H (2014) CFD: computational fluid dynamics or confounding factor dissemination? The role of hemodynamics in intracranial aneurysm rupture risk assessment. AJNR Am J Neuroradiol 35: 1849-1857

[13] Sugiu K, Martin J, Jean B, Gailloud P, Mandai S, Rüfenacht D (2003) Artificial Cerebral Aneurysm Model for Medical Testing, Training, and Research. Neurologia medico-chirurgica. 43: $69-72$

[14] Cardoso C, Fernandes C, Lima R, Ribeiro J (2018) Biomechanical analysis of PDMS channels using different hyperelastic numerical constitutive models. Mechanics Research Communications 90: 26-33

[15] Nunes L (2011) Mechanical characterization of hyperelastic polydimethylsiloxane by simple shear test. Mate Sci and Engin 528: 1799-1804

[16] Ribeiro J, Lopes H, Martins P, César MB (2019) Mechanical analysis of PDMS material using biaxial test", AIMS Materials Science, Issue 1, 6: 97-110 